\title{
Ueber Krystalle aus Taubenblut.
}

Von

Arthur Schwantke.

Assistent am mineralogischen Institut der Universität Marburg.

Mit Tafel II, Fig. 1-4.

(Der Redaction zugegangen am 16. Mai 1900.)

Die bisherige Kenntniss der verschiedenen Blutkrystalle beruht auschliesslich auf mikroskopischer Beobachtung. Die zahlreichen Publicationen über den Gegenstand geben zum Theil ein anschauliches Bild der charakteristischen Gestalten dieser Gebilde, es war auch möglich, durch Winkelmessungen unter dem Mikroskop und durch Beobachtung der optischen Erscheinungen für einzelne Krystalle das System und zum Theil auch die krystallographische Form zu ermitteln. Vorbildlich hierfür waren besonders die Beobachtungen v. Lang's ${ }^{1}$ ) über Krystalle aus dem Menschen-, Meerschweinchen-, Hunde-, Kaninchen- und Eichhörnchenblut, die später von Donogány²) wiederholt und fortgesetzt worden sind; diese und andere, wie die Untersuchungen Ewald's, ${ }^{3}$ ) zeigen aber zugleich die Grenze des für die mikroskopische Deutung überhaupt Erreichbaren, sobald es sich nicht um reguläre Krystalle handelt, deren Deutung auch unter dem Mikroskop möglich ist, wie

1) Sitzungsber. d. mathem.-naturw. Classe d. Kais. Akad. d. Wissenschaften, Bd. XLVI, II. Abth., Wien 1863, S. 85 f.

2) Zur Kenntniss der Hämoglobin- und Hämochromogenkrystalle. Math. és term. tud Éresitö 1892/93, 11, 262-287; mathem u. naturw. Berichte 1892/93, 11, 135-160. - Citirt nach d. Ref. in der Zeitschr. f. Krystallographie, 1894, 23, 499.

3) Polarispektroskopische Untersuchungen an Blutkrystallen. Zeitschrift für Biologie, 1886, 22 (N. F. 14), 459 f. 
sie von R. Panebiancol) aus dem Blute von Bombyx Mori beschrieben wurden. ${ }^{2}$ )

Herr Professor A. Kossel hatte die Güte, mir im Dezember vorigen Jahres Krystalle aus Taubenblut zur Untersuchung zu übergeben, die sich zu einer. eingehenderen makroskopischen Beobachtung eigneten.

Die Krystalle waren bis $2 \mathrm{~mm}$. lang und besassen für die Messung am Goniometer genügend spiegelnde Flächen. Die der Flüssigkeit entnommenen Krystalle waren weich und leicht zerdrückbar, nach dem Trocknen etwas fester. Um sie vor einer Zersetzung und Zerstörung zu bewahren, wurden sie in der Flüssigkeit vorsichtig an den Rand des Glases geschwemmt, mit einem Streifen Filtrirpapier aufgenommen und auf einem Uhrglase, das in durch Eis gekühltes Wasser gestellt war, durch Absaugen der anhaftenden Flüssigkeit mit dem Rande des Papiers getrocknet. Sämmtliche Beobachtungen am frischen Material wurden im physiologischen Institut der Universität Marburg in einem von Herrn Professor Kossel freundlichst zur Verfügung gestellten Zimmer bei einer zwischen 5 und $10^{\circ} \mathrm{C}$. liegenden Temperatur angestellt. Für die Messung am Goniometer wurden die Krystalle mit einer Mischung von Wachs und Vaseline aufgenommen und vor der Berührung mit der warmen Hand durch übergezogenen Handschuh geschützt. Es erfolgte zunächst die optische Untersuchung am Mikroskop, die auch nach der goniometrischen Messung wiederholt wurde, und in allen Fällen dem ersten Resultate entsprach und bewies, dass sich die Krystalle während der Dauer der Messung frisch erhalten hatten.

Die Krystalle erschienen im durchfallenden Licht dunkel-

1) Ueber Blutkrystalle. Rivista di Min. e. Crist. ital. di Panebianco, 1895, 14, 81. - Zeitschr. f. Kryst., 1897, 28, 198.

2) H. U. Kobert: Ueber das mikrokrystallographische Verhalten des Wirbelthierblutes, Leipzig 1900 (erw. Abd. aus Zeitschr. f. ang. Mikroskopie, V, 6-10), enthält eine Zusammenstellung der Litteratur und Beschreibung der verschiedenen Krystallarten, zum Theil auch auf Grund eigener Beobachtungen des Verfassers, ohne auf die genaueren krystallographischen Verhältnisse, namentlich das optische Verhalten, einzugehen. 
roth - dicke Krystalle auch nahezu undurchsichtig - und zeigten nur sehr schwachen Pleochroismus, übergehend in ein etwas helleres Roth. Demgemäss waren sie (den Beobachtungen Ewald's [loc. cit.] zufolge) als Oxyhämoglobin aufzufassen, was von Herrn Professor Kossel durch spektroskopische Untersuchung bestätigt werden konnte. Die Auslöschung im parallelen polarisirten Licht erfolgte parallel der Kante $\mathrm{m} / \mathrm{m}$ (Taf. II Fig. 1 und 2). Im convergenten Licht erwiesen sich die Krystalle als einaxig, indem beide m-Flächen kein Axenbild, sondern das Verhalten parallel der Axe geschnittener Platten zeigten; die Beobachtung durch beide Flächen war allerdings wegen der tafeligen Gestalt der meisten Krystalle nur in wenigen Fällen möglich. Schnitte senkrecht zur Axe waren von den Krystallen nicht $\mathrm{zu}$ gewinnen, es gelang aber in zwei Fällen Krystallsplitter zu erhalten, die im convergenten Licht bei der Drehung des Tisches gerade über das Gesichtsfeld hingehende Balken zeigten. Breit gedrückte Krystalle zeigten undulöse Auslöschung und wurden nach einiger Zeit isotrop.

Die krystallographische Untersuchung ergab die Zugehörigkeit der Krystalle zur sphenoidischen (tetraëdrischen) Hemiëdrie des tetragonalen Systems. Der Winkel $\mathrm{m} / \mathrm{m}$, sowie der Winkel der oberen p-Flächen $\mathrm{zu} \mathrm{m}_{2}$ und der der unteren zu $\mathrm{m}_{1}$ betrug $90^{\circ}$, der Winkel der oberen p-Flächen (zu einander bezw. $\mathrm{zu} \mathrm{m}_{1}$ ) war gleich dem der unteren (zu einander bezw. $\mathrm{zu} \mathrm{m}_{2}$ ). Die Krystalle sind also Combinationen eines tetragonalen Prismas $\mathrm{m}=(110)$ mit einem Sphenoid $\mathrm{p}=+(111)$.

In Folge der unvollkommenen Signale waren die Messungen einer ziemlich beträchtlichen Schwankung unterworfen. Es zeigte sich dabei aber - dem tetragonalen System entsprechend - ein Unterschied zwischen den rechten Winkeln und den Winkeln der p-Flächen, indem die letzteren in weit höherem Maasse, ungefähr innerhalb einer Grenze von 2 Graden, schwankten, was durch die unvollkommene Flächenbeschaffenheit allein nicht erklärt werden kann. Ein Unterschied der oberen von den unteren $\mathrm{p}$-Flächen zeigte sich dabei nicht.

Als wahrscheinlichster Mittelwerth der gemessenen besten 
8 Krystalle darf der Winkel $\mathrm{p}: \mathrm{m}=(111):(110)=31^{\mathrm{a}}$ (Normalenwinkel) gelten, was insofern mit der Messung gut übereinstimmt, als die zuverlässigste Messung aus der Reihe der Beobachtungen von $p: p=(111):(\overline{1} \overline{1} 1)$ einen Werth von $118^{\circ} 06^{\prime}$ ergab, was einem Werth von $\mathrm{p}: \mathrm{m}=30^{\circ} 57^{\prime}$ entsprechen würde. Aus dem Winkel $31^{\circ}$ berechnet sich das. Axenverhältniss $\mathrm{a}: \mathrm{c}=1: 1,175$ und der Winkel $\mathrm{p}: \mathrm{p}=$ $(111):(1 \overline{1} \overline{1})=108^{0} 18^{\prime}$, gemessen $106^{\circ} 39^{\prime}$.

Die meisten Krystalle zeigen nicht die ideale Form der Fig. 1 und 2. Sie sind in der Regel der Gefässwand anliegend gewachsen und zeigen dann an der aufliegenden Seite eine höchstens $m$ vicinale oder ganz schief gelegene Fläche, die ziemlich eben und glänzend aussieht, aber am Goniometer unschwer als Scheinfläche erkannt wird. Die Krystalle erhalten dann eine unsymmetrisch erscheinende Gestalt, wie sie Fig. 4 veranschaulicht. Freigewaschene Krystalle zeigen in der Regel eine ungleichartige Ausdehnung der p-Flächen wie Fig. 3 (die wie 4 mit horizontaler c-Axe gezeichnet ist).

Charakteristisch sind an den Krystallen kleine Pyramiden, die sich auf den Prismenflächen am spitzen Ende erheben, wie Fig. 2 zeigt. Sie werden gebildet von einer Fläche $P$, die mit. der gegenüberliegenden $p$-Fläche einspiegelt $\left[\mathrm{P}_{1}\right.$ mit $\left.\mathrm{p}(111)\right]$, sowie von zwei anderen Flächen $\pi$, die sehr unvollkommen ausgebildet sind, gerundet und gestreift, so dass eine nähere Bestimmung am Goniometer unmöglich ist, die aber im Allgemeinen auf der Prismenfläche senkrecht und mit den pFlächen des betreffenden Krystallendes parallel zu sein scheinen. Die Erscheinung wurde ziemlich häufig und zum Theil auf beiden Prismenflächen, wie in Fig. 2, beobachtet. Beide Pyramiden würden einem Sphenoide ohne Prisma entsprechen, es würde also eine parallele Fortwachsung der Krystallsubstanz vorliegen. ${ }^{1}$ )

Die Krystalle hielten sich, im Gläschen bei kalter Temperatur aufbewahrt, mehrere Wochen. Nach 3 Monaten waren

1) Ergänzungszwillinge der sphenoidischen Tetartoëdrie anzunehmen, liegt jedenfalls kein Grund vor, so lange die Prüfung durch Aetzfiguren, wie am vorliegenden Material, unmöglich ist. 
sie verändert, hart, spröde und fast undurchsichtig, nur bei sehr geringer Dicke noch mit rother Farbe durchscheinend, aber im polarisirten Licht völlig isotrop. Die Flächen waren dagegen durchaus glänzend und spiegelnd geblieben, so dass die Krystalle für die goniometrische Messung noch geeignet waren. Die nochmalige Messung des einen Krystalls ergab ein mit den am frischen Krystall gemessenen Werthen übereinstimmendes Resultat.

Auch die durch v. Lang (loc. cit.) beschriebenen Krystalle aus Meerschweinchenblut zeigten eine sphenoidische Gestalt, indessen wurde die Zugehörigkeit zum rhombischen System durch das optische Verhalten bewiesen und durch Donogány (loc. cit.) bestätigt. Bojanowski ${ }^{1}$ ) bildet sphenoidisch aussehende Krystalle aus dem Blute des Raben ab (Taf. XXX Fig. 12) und bemerkt (S. 335), dass er ähnliche Krystalle aus Taubenblut erhalten habe. Wie weit diese mit den oben beschriebenen übereinstimmen, ist daraus nicht zu schliessen.

Es erscheint wünschenswerth, dass es gelänge, auch die Krystalle anderer Blutarten, sowie aus demselben Blute die Krystalle der anderen Modificationen oder Verbindungen des Hämoglobins zu untersuchen. In physikalischer Beziehung scheinen die Blutkrystalle die interessanten Eigenschaften anderer eiweissartiger Körper zu theilen. Das Quellungsvermögen und die Fähigkeit, Lösungen fremder Stoffe aufzunehmen, wie es von den Albuminen bekannt ist, ${ }^{2}$ ) scheint, schon den Beobachtungen Reichert's ${ }^{3}$ ) zufolge, auch den Blutkrystallen eigen zu sein. Vielleicht ist auch die oben beschriebene Weichheit der Krystalle und die Schwankung in den Winkelwerthen

1) Beobachtungen über die Blutkrystalle. Zeitschr. für wissensch. Zoologie, 1863, XII, $312 \mathrm{f}$.

2) Vergl. u. A. Schimper, Ueber die Krystallisation der eiweissartigen Substanzen. Zeitschr. für Kryst., 1881, 5, 131f. und Wichmann, Ueber die Krystallform der Albumine. Zeitschr. für physiol. Chemie, 1899, Bd. XXVII, S. $575 \mathrm{f}$.

3) Beobachtungen über eine eiweissartige Substanz in Krystallform. Müller's Archiv für Anat. u. Physiol., 1849, $197 \mathrm{f}$. 
(3)

Brought to you by | Purdue University Lib Authenticated

Download Date | 6/19/15 5:17 PM 


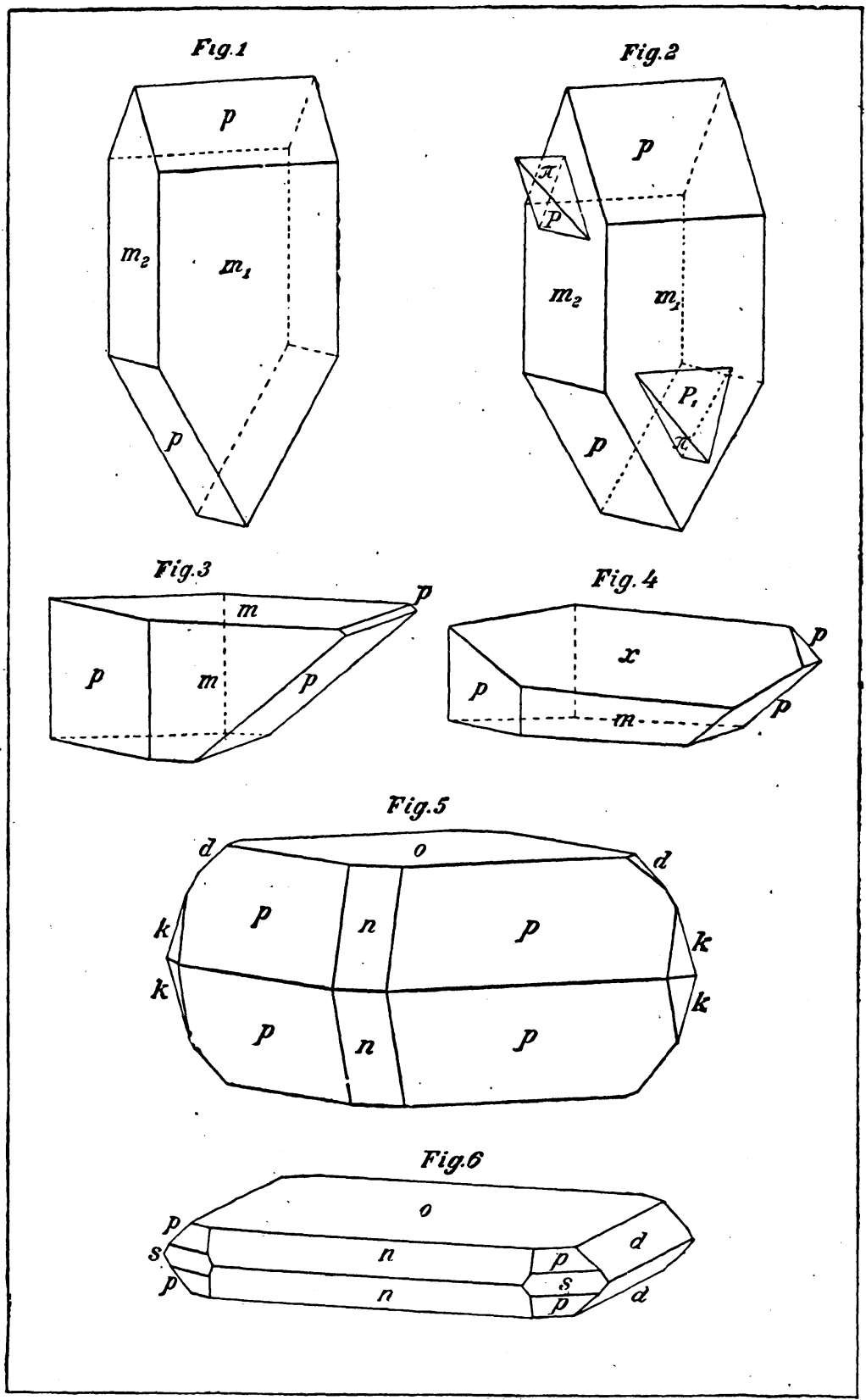

A. Schwantke del.

Hoppe-Seyler's Zeitschrift für physiologische Chemie, Band XXIX.

Verlag von Karl J. Trübner Strassburg.

Zu « Schwantke, Ueber Krystalle aus Taubenblut *. 
hierdurch zu erklären. Zur näheren Untersuchung ,würde es allerdings eines Materials bedürfen, wie es nur unter besonders günstigen Umständen $\mathrm{zu}$ erhalten sein wird. Die oben beschriebenen Krystalle waren zu weiteren Versuchen nach dieser Richtung hin ungeeignet. Jedenfalls verdienen aber alle diese Körper auch das Interesse des Krystallographen in hohem Grade.

Herrn Professor A. Kossel sei auch an dieser Stelle für die gegebene Anregung und freundliche Unterstützung herzlicher Dank ausgesprochen!

Marburg, den 15. Mai 1900.

\section{Tafelerklärung.}

Fig. 1. Ein Krystall des Oxyhämoglobins aus Taubenblut in tafeliger Ausbildung nach der einen Prismenfläche. Combination $p+(111)$, $m(110)$.

Fig. 2. Dieselbe Combination mit den Prismenflächen im Gleichgewicht. Auf den letzteren die einer parallelen Fortwachsung entsprechenden von den Flächen des Sphenoids gebildeten Pyramiden.

Fig. 3. Ein frei gewachsener Krystall derselben Combination mit ungleich entwickelten p-Flächen.

Fig. 4. Ein unvollkommen ausgebildeter Krystall derselben Combination, gewachsen auf der Scheinfläche $x$.

Fig. 3 und 4 sind mit horizontaler c-Axe gezeichnet.

\section{Zur folgenden Abhandlung:}

Fig. 5. Ein Krystall des Histidinmonochlorids. Combination: p (111), o (001), n (101), $\mathrm{k}(121), \mathrm{d}$ (012). Axenverhältniss: $\mathrm{a}: \mathrm{b}: \mathrm{c}=$ $0,76665: 1: 1,71104$.

Fig. 6. Ein Krystall des Histidindichlorids. Combination: o (001), $n$ (101), $d$ (012), $p(111), s(110)$. Axenverh.: $a: b: c=0,76537: 1: 1,77516$. 